\title{
Children and Theory of Mind; Comparison between Children's Ability to Understand Trying Actions and Pretend Actions in a Behavior Model Analysis
}

\author{
Besjona Dede
}

Doctoral Student, Department of Psychology and Pedagogy, Faculty of Social Sciences University of Tirana

\author{
Doi:10.5901/jesr.2015.v5n1s1p19
}

\begin{abstract}
Children who have just reached 2 years old do engage in activities with symbolic elements. Developmental psychologist argue that this is a cognitive developmental feature connected to mind processes development. Some of them discuss about using indented/pretended activities as fact of child' understanding. The research involved 20 children 18 to 25 months old and 25 children 25-36 months old. Children were applied the behavioral protocol of imaginary and intentional behavior. Children's reactions to these actions is observed, measured and assessed using behavioral protocol activities. The purpose of this study is to explore the ability of children 20 to 36 months to understand the intentional actions against symbolic/pretended actions. This study is theoretically based on cognitive development theories in early childhood, focusing on the use of symbolic functions that enable children involvement in the play. Certain elements of the study were stimulated by new theories on the cognitive functioning of representative schemes, ToM and comprehension skills during early childhood. The results of the study show a tendency of children to understand the purpose of the two behavioral structures answered in accordance with the typology behavior: after the demonstration of intentional models children react intentionally and after the demonstration of pretend actions children reacted with imaginative / symbolic actions. The results over the ability to distinguish intention from pretend is then discussed in the Theory of Mind perspective.
\end{abstract}

Keywords: Theory of Mind (ToM), cognitive structures, pretend play, early childhood development, intentional models, pretend models.

\section{Introduction}

The "behave if" play is such a common activity for children who pass in the second year of life that it is not impossible to be noticed by adults. Their commitment to this form of play is made possible by a set of features associated with motor development, physical, social and emotional. Ability to act, distinguish, claime are necessarily influenced by the level of development of cognitive features of this age.

The term "theory of mind" is used to refer to the development of concepts of mental activity in children. However, theory of mind is more than just a collection of concepts. The theory includes a coherent framework to organize the facts and to make predictions. Theory of mind (Bjorklund, 2005,p.255) includes recognition of different categories in mind as dreams, memories, imagination, and other beliefs that have a frame of causal explanation about the actions of other people.

One aspect of theory of mind in early childhood is related to knowledge of child development; aspects of thinking and implementation of actions and other issues related to cognition as metacognition and representation (Flavell, Green, \& Flavell, 1998; Schwanenflugel, Henderson, \& Fabricius, 1998 cit. by Bjorklund, 2005 p.256).

Generally there are two groups of researchers discussing about the theory of mind (Wellman et.al, 200, p. 659). Members of the first group argue that children 3 years old (and probably younger) have the same basic abilities as older children but the demands of tasks, issues and complex information processing limitations prevent them from displaying these abilities in many situations.

Members of the second group suggest that there is a real conceptual change during the preschool years and there are age-related differences in the performance of actions "false belief". So, they are the result of "real change in the concept of children on the individual" (Wellmanet. al., 2001 p. 671)

Cognitive development is part of the holistic development of the individual. Basically it is believed that cognitive development processes are a potential combination of genetic and environmental stimuli. There are two different approaches to cognitive development during the last decade: The first is the increasing emphasis on the biological basis of development and the second position is the increasing emphasis on the social construct of cognition; perspective that the way children learn to think is run by the culture. 
Socialcultural psychologists believe that the way we develop, especially the way we think is the primary function of the social and cultural environment in which we grow. This view emphasizes the fact that people think in different ways about the fact that all we have in common the fact we're human being. The universal point of view on cognitive development of Jean Piaget emphasize the cognitive features, the aspects of development that characterize the development in every child. In this attitudes' discussion the conclusion is that cognitive skills are defined by innate traits but the environment determines the development potential of these features.

Thus, according Piaget, the cognitive development passes through several stages. At the age of 2-7 years old children are in the stage of operational thinking. During this stage, children learn symbols that represent objects, play with dolls, develop imagination, imitate the actions of adults (Orhan, 2005, p. 61). The main principle is related to the child, not as a human being that simply imitate itself (J. Piaget, 1976, p. 14). Studies in recent years on child development support the idea of association between cognitive development and symbolic game during early childhood. Based on the classical theory of Piaget, on cognitive development in early childhood children begin using symbolic functions. Thus, according to Harris and Kavanaugh, (2006 cit. Rakoczy ,Tomasello, p.558), after the implementation of some experiments proved that around the age of 2 years children begin to understand and follow the pretend actions by presenting them in a pretend scenario. As related to cognitive ability, Liliard (1998, p.20) has noticed that pretend play involves negotiation between parties with different views, the representation of objects in two ways (real and pretend), role play, all actions that suggest that children who engage in these activities mental representation skills. In the study of Rakoczy \& Tomasello, (2006 p.557-564) children 22 months made similar general patterns reaction, although in a much easier way: Generally these children responded significantly to both types of models, revealed by the fact that each type of conclusive answers was performed significantly more often in the same conditions compared with the opposite conditions.

The concept of theory of mind is developed based on the theory explaining the development of the mind. The theory presented by Simon Baron-Cohen (1995 cit by Bjorklund, 2005, p. 255) relates to the interaction of four separate modules from each other that include reading the mind and development during infancy and early childhood. The earlier module is intentional investigator (ID) that interprets moving objects based on aim or purpose. The second module is of the direction of the eyes (EDD) has three functions related to each other: the presence of visibility investigating to determine the presence of viewing or viewing as a stimulus to determine whether visually refers to the individual or another and interferes thought that when the eye sees something, then that individual is seeing that. These two modules zhvilllohen from birth until the 9th month of life. Module tretëështë divided attention mechanism (SAM) which includes three modes of interaction and representation (achievement of deduction) Simon Baron-Cohen (1995 cit by Bjorklund, 2005, p. 257). This module takes place in the month of 9 to 18 of life. And the last module of the Theory of Mind (Tomm) qëështë substantially similar to real-desire reasoning and takes place between the ages of 18-48 months.

Rakoczy, Tomasello and Striano (2004, f.388-399) have challenged the theory of behavior as in the claim that children under four years of age do not understand the claim as a form of targeted action. They pretend that younger children already understand the claim as a form of action with specific purpose demeanor as different from other forms of behavior as though they do not understand the epistemic structure of claim, although difficult to accomplish tasks complex.

In a set of experiments conducted by Rakoczy and Tomasello, (2006, p. 557-564) children 36 months showed very clearly that they understand the claim and effort in such models: models attempt after they performed actions to realize real trying often commented (eg, "I can not do this ") but after models claim they did not care about the real consequences of their actions ( $p . s h$ if there tis water in the holder). Children 26 months showed the same patterns, most models attempt to realize real action or behavior, attempts have and rarely showed by the pretneder. Following models claim they generally performed feedback and trying that claim could lead to the conclusion that the effort may be impossible action at this age. One more important case is that after trying models in children 26 months more answers fail to meet the criteria to be trying and pretending.

In a third study Racoczy et.al (2009, p.61-69) similar patterns were presented to children 3 years old but not in a conditional imitation game. For encroached upon the practical conditions of the situation are determined to encourage more productive and decisive response by introducing additional objects as working tools and toys that can be used in operations and true claimant. So in this study and 3 years old children performed significantly more correct than incorrect answers after any kind of model.

These findings were also added to the credibility of the data field of the development of claim: Harris and Kavanaugh (1993) for example found consistently that around the age of 2 years children start to become proficient in understanding and pursuing the pretendurs scenarios undetected. An additional explanation could be that children may 
understand what an adult might have done in this model but failed to show sufficient signals in their reactions to achieve the behavior rating scale claimant.

In their theory Nichols. S \& Stich.S (2000) claimed representations are kept in a separate mental work, a Possible World Box, that is part of the architecture of the human mind. Representations in the Possible World Box is likely to have the same content as beliefs. Mental representations have the same "code" representation as beliefs and representations in Possible World Box processed from the same update mechanisms and setting (conclusion) that operate on real beliefs. This model also sets a scenario processor included in the decoration of the facts that were there. The authors assert that the behavior seen in pretend play is motivated not by a "desire claim" but from a real desire to act in a way that fits the description built by the Possible World Box. The authors defend the idea that this structure can accommodate the central features of the actions shown in the pretend examples and that alternative structures or can not accommodate or fail to address some pretend features.

Thus, the pretend episodes typically begin with an initial condition or set of conditions which are basic principles on that will be pretend. Pretended behavior is initiated by the pretender in a initiator condition (if he / she who initiates) and must indicate which is the initial condition and decide on the succession if the is other condition or pretender that initiates the pretend action. If the pretender decides to countinue, the his / her cognitive system should begin to generate thoughts and actions appropriate to the authenticity of the pretended condition.

Then the determination often plays an important role in meeting the details of what happens during the pretend action. Starting from the initial conditions and then the perceptions, existing knowledge, the memory of what happened in the episode and no doubt from many other sources the pretender is able to chart definitions on what is happening in the claim.

Beyond determining processing in children and adults process and claim scenarios in ways that are not definitive. In some circumstances it is a matter of filling in history by the scenario. Perhaps the most visible evidence on the claim is that the claimant makes the actions that are appropriate for the claim.

Episodes can last claim in different time periods. When an episode is finished claimant typically not claiming implementing activities and events that have occurred in the context of the claim and are a limited effect on the mental state post claimant. One obvious way in which they appear nIn limited form is when claimants do not believe that the alleged events are happening in reality. Moreover, as Leslie (1987) noted, even the youngest children do not believe that bananas are mobile. And as more adults. For even more during the course of themselves claim, the claimant believes it really is typically distinct from what he believes in the case of claiming episodes context. The belief system of contenders is not completely isolated from the content of the claim. After an episode people typically claim individuals typically have accurate beliefs about what happens in the episode pretender; they remember the case of claim.

\section{Methodology}

\subsection{Research question}

The research question of the study is : Does children 18 to 36 months have the ability to distinguish actions as intentional or symbolic?

\subsection{Participants}

The sample consisted of 20 children 18 -24 months old and 25 children 25-36 months old; The children selected attend public day care and education institutions (crèches in Tirana city). The Groups of children were selected at random at 3 day care centers. The selection criteria were age and gender. The selection ratio of the age and gender aimed to respect gender and age ratios in the general population of children that frequent the day centers in Tirana. The selection of institutions have all been conducted based on criteria of homogeneity of social factors.

\subsection{Application}

There was applied a model based on the protocol used by Hannes Rakoczy and Michael Tomasello (2006) by a trained specialist. Activities and games used in this procedure were selected from the education program that is implemented in public kindergartens based on group age. There were presented two models of action: if it was eating / trying to eat; pour pretending / trying to pour. Each child was presented two action models of trying and two action models of pretending by 
the same theme. The order of operations and the definition of objects of the same subject to trying and pretending actions change from case to case. The order of topics within the same sequence was the same. In the beginning the tester specialist and child played freely, the tester did some simple actions, two pretending actions with an object whatever, two pretend actions and asks the child to do the same action. Then realized the first testing session, which consists of two models of actions. The procedure followed by the second session of the trying and pretend the extra phase and the second testing session. During the application protocol a specialist based on coding conducted the behavior classification.

\subsection{Procedures and preparatory actions}

Simple actions preparatory of pretend and trying (1) dig a whole (with a new object, (2) behave if makes a call (with the same object) (3) trying to make music with piano children (by pressing a button that does not work) 2 . The first trying and pretending section with additional action: (1) behave if someone is brushing the teeth, (2) behave if it is making a shower (3) attempting to open a container (using pliers), (4) trying to open a second container (then using pliers), (5) act if it is drinking and as it is opening a bottle of fruit juice, (6) cutting some dough with pliers, (7) behave if it is cooking something.3. session of the first test (for half of the children the pretending behavior, the other half the trying behavior) 4 . The second trying and pretend session with additional action, (1) behave if someone combs the hair, (2) trying to open a box, (3) behave it is washing something, (4) tries to write (with a pen that has a lid) .5. The second testing session (for half of the children the pretending behavior, the other half the trying behavior).

Each coding behavior was classified into one of five categories: "inferential pretend" when the child performs an action in accordance with the thematic contender, that goes beyond what the tester performs; "simple pretend" when the child performs what seems like a clear contender. Conversely, responses were coded as "inferential trying" when the child discovers from an action / word recognition that the goal was to implement a successful action or as "simple trying" when the child perform something that looks like a clear example of a trying behavior. Finally, the category "unclear" answers that do not meet the above categories.

\subsection{Validity and ethical principles}

The using standard procedures has minimize subjectivity in applying the protocol from the testers and in data interpretation. The procedure is carried out by a tester/specialist who is instructed to use the same standards applicable to each behavioral sequence of each child. The tester is supervised. The behavioral coding is performed by an appraiser who is instructed and trained to follow the same behavior as indicators of ability. To increase the validity of coding is used a double check procedure. The institutional permission and the informed consent is obtained in advance by the institutions and parents. During the study the ethical and moral principles have been respected. Confidentiality was respected during the work with children.

\section{Results}

At the end of the study showed that from 20 children 18- 25 months during the intentional stimulation behavior model 4 of them follow "inferencial pretending", 3 show "simple pretend", 5 showed "inferential intention", 7 show "simple intention" and the behavior of one child was classified "not clear". While during pretending stimulation model behavior 6 children show "inferencial pretending", 5 children showed "simple pretending", 3 showed "inferential intention", 4 show "simple intention/trying" and the behavior of two children was classified "not clear ". While the behavior of 25 children 2536 months showed that from during the intentional stimulation behavior model 3 of them follow "inferencial pretending", 4 show "simple pretend", 6 showed "inferential intention", 7 show "simple intention" and the behavior of 5 children was classified "not clear". While during pretending stimulation model behavior 7 children show "inferencial pretending", 3 children showed "simple pretending", 3 showed "inferential intention", 3 show "simple intention/trying" and the behavior of 5 children was classified "not clear".

\section{Discussion}

Thus studies in the theory of mind provide complete data than 3 years old children understand the target pretend structure as a specific form of action and game different from other forms of behavior though. In contradiction to the 
theory of "if behavior", these data indicate that children at smaller age have at least the ability to understand the hidden elements of the pretended or intentional actions. The data for children 2 years old are really unclear. In imitative character studies fail to show children the more correct than incorrect responses after pretend models. This may be a consequence of the conceptual problem that children can not understand the true structure of the pretend actions in target.

Another explanation may be the result of unclear instructions or negative ones. One possibility could be that the problems in the implementation of the guidelines problems occur due to improper submission after pretending models; although they perceive action model presuming they see that the object can be used to perform the action in reality and were unable to overcome the tendencies of "superiority", etc. Another source could be that children misunderstand practical elements of the situation, so they understand the actions modeled as claims and deliberately misinterpret the model as pedagogical aspect.

This option becomes more plausible by the fact that after pretending models many children 2 years old give many answers that do not meet either criteria or attempt to claim.

According to the research issues examined in the present study, specific results show that children 20 to 36 months have the ability to distinguish intentional actions symbolic ones. Children 25-36 months have more ability to distinguish intentional actions with symbolic ones under the stimulus of the respective models; children 20-25 months understand the aim' actions and follow the presented models but find it difficult to improvise beyond the model to introduce and express in words the quality of the acts performed. In a detailed comparison between reactions compatible and not compatible after the presentation of each model, the children presented more compatible responses than noncompatible ones after the presentation of the model. So, the symbolic play influence the cognitive development and the cognitive skills according to the development stage influence the pretend play performance in early childhood. While on the findings in this study show that children 3 years of age but probably sooner have the ability to distinguish the actions purposiveness of the self and others; Well they have a theory of mind.

\section{References}

S Goldstein \& J Naglieri, Encyclopedia of Child Behavior and Development, Springer Science+Business Media LLC, 2011, f. 522-523.

A Lillard, Playing with a theory of mind, In O. N. Saracho \& B. Spodek Multiple perspectives on play in early childhood, Albany: State University of Neë York Press, 1998 f. 11-33.

Z Orhani, Psikologjia konjitive, Ada, 2005, f.61, 262-263.

R Shelov and R Hannermann, Caring for your baby and young child: Birth to 5, American Academy of Pediatrics, 2004 www.cdc.gov/ actearly

D Bergen, The Role of Pretend Play in Children's Cognitive Development, ERIC Clearinghouse on Elementary and Early Childhood Education Champaign IL Miami University, Volume 4 Number 1, 2002.

M Robinson, Child Development 0-8, A Journey through the Early Years, Open University Press, NY, 2008, f.157

J Piaget, The child conception of the world, Littlefield, Adams and CO, Totoëa New Yersey, 1976, f.14

S Johnson, The nature of cognitive development, Trends in Cognitive Sciences, Vol.7, No.3, Mars 2003 f.102-104.

Hannes Rakoczy*, Felix Warneken, Michael Tomasello, Young children's selective learning of rule games from reliable and unreliable models, Cognitive Development 24 (2009) 61-69

Rakoczy H, Tomasello M, Striano T. Young children know that trying is not pretending: a test of the "behaving-as-if" construal of children's early concept of pretense. Dev Psychol. 2004 May;40(3):388-99.

H Rakoczy and M Tomasello, Two-year-olds grasp the intentional structure of pretense acts, Developmental Science 9:6 (2006), f. 557564.

Kelly, R., Hammond, S., Dissannayake, Ch., Ihsen, E., The relationship between symbolic play and executive functions in young children, Australiasia Journal of Early Childhood, pp 21-28;

Bjorklund. David, "Children's Thinking; Cognitive Development and Inidividual Differences", Bot. i IV; Wadsworth, 2005

Wellman HM, Cross D, Watson J. Meta-analysis of theory-of-mind development: the truth about false belief Child Dev. 2001 MayJun;72(3):655-84. 
